\title{
Effective stress concept for mechanical modeling of clays under different environmental conditions
}

\author{
Angelica Tuttolomondo ${ }^{1}$, Alessio Ferrari ${ }^{1}$ and Lyesse Laloui ${ }^{1}$ \\ ${ }^{1}$ École Polytechnique Fédérale de Lausanne (EPFL), School of Architecture, Civil and Environmental Engineering (ENAC), Laboratory \\ for Soil Mechanics (LMS), EPFL-ENAC-LMS, Station 18, Lausanne CH-1015, Switzerland
}

\section{Introduction}

Clays (especially if active) may exhibit different mechanical responses depending on their environmental conditions (e.g., Di Maio 1996; Manca et al. 2016). Due to this, advanced modeling of the mechanical behavior of clays must pay particular attention to the adopted: (i) stress variables, (ii) strain variables, and (iii) stress-strain relationships. In the context of continuum mechanics, a suitable effective stress framework can allow converting a representative elementary volume (REV) of real medium with $\mathrm{m}$ mixtures (solid substances and fluids) subject to $\mathrm{n}$ actions (total stress changes and environmental changes) into a mechanically equivalent continuum medium subject to a change in effective stress. In this study, a suitable effective stress concept for clays is presented, and the benefits of using it are demonstrated. For this purpose, first, based on recent physical-chemical studies of water-clay mineral interactions, the different types of ions and water in clays are recalled. Secondly, a thermodynamic-geochemical integrated approach is utilized to redefine the effective stress concept taking these findings into account. By analyzing experimental data taken from the literature, implications of using the proposed effective stress concept are finally explored. In this regard, among others, the uniqueness of the critical or residual failure envelope regardless of the chemical composition of the pore water or the saturation state is noteworthy.

\section{Effective Stress Concept and Mechanical Modeling Implications}

Figure 1 provides terminology for water and ions in the presence of clay particles (Tuttolomondo et al. 2020). Cations dissociated from the clay mineral surface are named non-movable ions; they can extend for more than $1 \mathrm{~nm}$ (Tournassat et al. 2009), up to $50 \mathrm{~nm}$ according to (Terzaghi et al. 1996). All other cations or anions are called movable ions. As a result of its structural peculiarities (Tuttolomondo et al. 2020), water within 1 $\mathrm{nm}$ from the mineral surface is classified as solid water; water beyond $1 \mathrm{~nm}$ is classified as liquid (pore) water.
Thus, particular emphasis must be placed on recognizing the possible presence of movable and non-movable ions in liquid water. The diffuse layer and bulk water consist only of non-movable ions and movable ions, respectively.

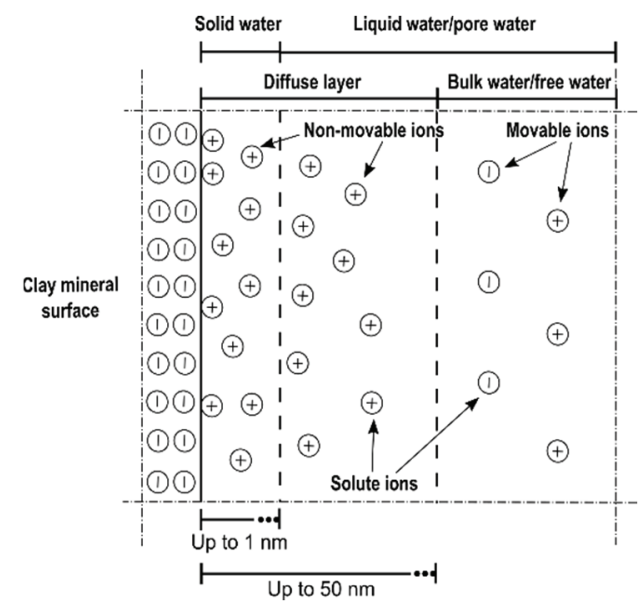

Fig. 1: Definition of water and ions in clays (redrafted from Tuttolomondo et al., 2021)

For unsaturated soils, the effective stress variable would take into account three different mixtures - solid mixture, liquid water, and gaseous solution (e.g., Nuth and Laloui (2008a)). By referring to unsaturated states and following a thermodynamic-geochemical integrated approach (like in Tuttolomondo et al. 2021), the effective stress variable for unsaturated clays can be expressed as follows:

$$
\sigma_{i j}^{\prime}=\sigma_{n e t, i j}+S_{r}\left(s_{m}-s_{s, e}\right) \delta_{i j}
$$

where $\sigma_{n e t, i j}=\sigma_{i j}-u_{g} \delta_{i j}$ is the net stress tensor $\left(\sigma_{i j}\right.$ : total stress tensor, $u_{g}$ : gas pressure, $\delta_{i j}$ : Kronecker delta), $s_{m} \delta_{i j}=\left(u_{g}-u_{w}\right) \delta_{i j}$ is the matric suction stress tensor ( $u_{w}$ : measured, or externally imposed, water pressure), $s_{s, e} \delta_{i j}$ is the effective solute suction stress tensor, and $S_{r}$ is the degree of saturation. The variable $s_{s, e}$ is the difference between solute suction in the pore water and

\footnotetext{
* Corresponding author: angelica.tuttolomondo@epfl.ch
} 
bulk water. The former depends on both movable and non-movable ions, and an analitcal procedure for its determination is developed; the latter only on movable ions. Relationships describing the evolution of $s_{m}$ and $s_{s, e}$ during the stress path of interest are essential in the newly developed framework.

Figure 2 shows the residual shear strength data of Ponza bentonite (plasticity index with distilled water equal to $320 \%$, limit liquid with distilled water equal to 390\%; Di Maio (1996) and Di Maio et al. (2004)), saturated with different solutions (Di Maio 1996), reinterpreted according to Terzaghi's effective stress (Fig.2a) and using the proposed definition (Fig. 2b) (Eq.1 for saturated states yields $\left.\sigma_{i j}^{\prime}=\sigma_{i j}-\left(u_{w}+s_{s, e}\right) \delta_{i j}\right)$ (cation exchange capacity equals $74 \mathrm{cmol} / \mathrm{kg}$; fraction of non-movable cations within the pore water equals 0.15 , (Tuttolomondo et al., 2021)). The latter framework enables better interpretation.

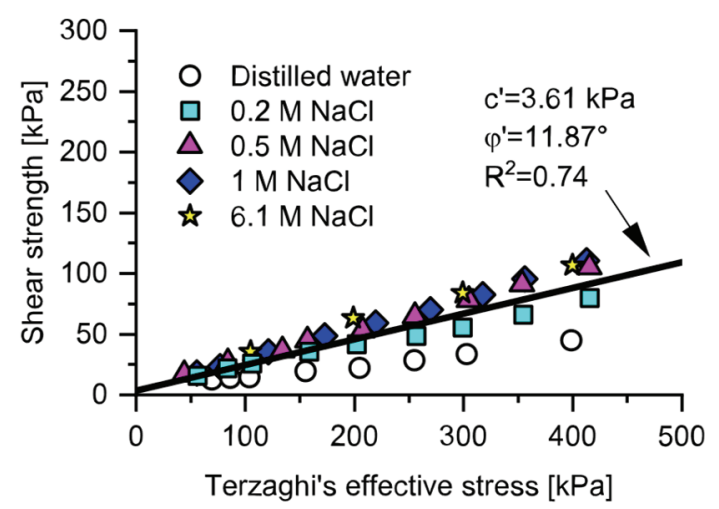

(a)

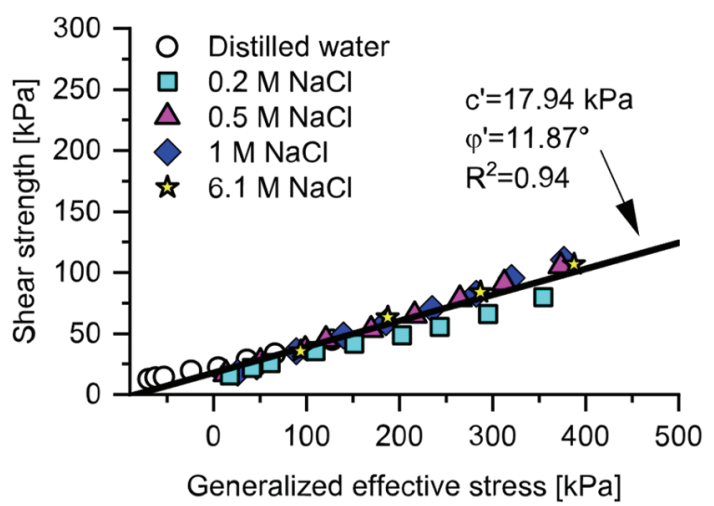

(b)

Fig. 2: Shear strength data with varying salt concentrations (experimental data from Di Maio, 1996) (redrafted from Tuttolomondo et al., 2021)

Figure 3 shows $s_{m}$ and $s_{s, e}$ at varying $S_{r}$ for compacted scaly clay from Sicily (liquid limit in the range of $60-64 \%$, plastic limit in the range of $20-26 \%$ ), at failure (Rosone et al. 2016). Soil water retention data are modeled according to Nuth and Laloui (2008b). Effective solute suction data are back-predicted based on shear strength and retention data. Using the developed analytical approach, back-predicted data are satisfactorily modeled.

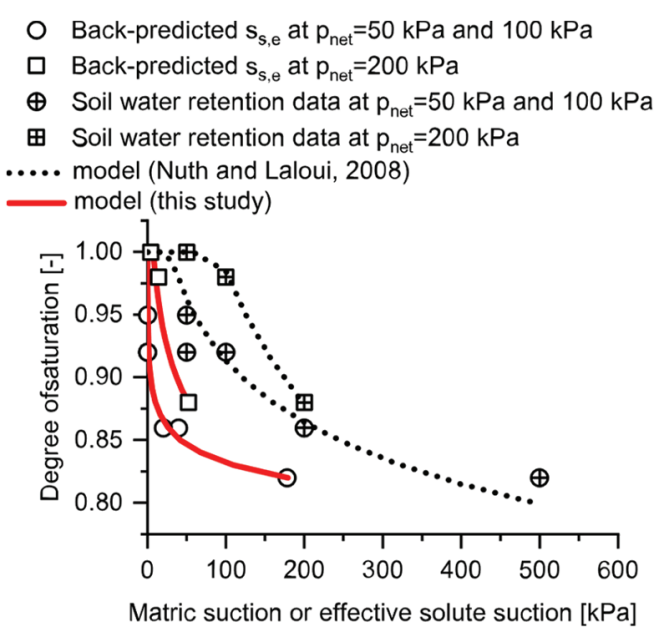

Fig. 3: Evolution of $\mathrm{s}_{\mathrm{m}}$ and $\mathrm{s}_{\mathrm{s}, \mathrm{e}}$ at varying $S_{r}$ (experimental data from Rosone et al., 2016) (pnet: mean net stress)

\section{References}

1. Di Maio, C. (1996). "Exposure of bentonite to salt solution: osmotic and mechanical effects". Géotechnique, 46(4), 695-707.

2. Di Maio, C., Santoli, L., and Schiavone, P. (2004). "Volume change behaviour of clays: the influence of mineral composition, pore fluid composition and stress state". Mechanics of Materials, 36, 435-451.

3. Manca, D., Ferrari, A., and Laloui, L. (2016). "Fabric evolution and the related swelling behaviour of a sand/bentonite mixture upon hydro-chemomechanical loadings". Géotechnique, 66(1), 41-57.

4. Nuth, M., and Laloui, L. (2008a). "Effective stress concept in unsaturated soils: Clarification and validation of a unified framework". International Journal for Numerical and Analytical Methods in Geomechanics, 32(7), 771-801.

5. Nuth, M., and Laloui, L. (2008b). "Advances in modelling hysteretic water retention curve in deformable soils". Computers and Geotechnics, 35(6), 835-844.

6. Rosone, M., Airò Farulla, C., and Ferrari, A. (2016). "Shear strength of a compacted scaly clay in variable saturation conditions". Acta Geotechnica, 11, 37-50.

7. Terzaghi, K., Peck, R., and Mesri, G. (1996). "Soil mechanics in engineering practice". John Wiley \& Sons.

8. Tournassat, C., Chapron, Y., Leroy, P., Bizi, M., and Boulahya, F. (2009). "Comparison of molecular dynamics simulations with triple layer and modified Gouy-Chapman models in a $0.1 \mathrm{M} \mathrm{NaCl}$ montmorillonite system". J Colloid Interface Sci, 339(2), 533-541.

9. Tuttolomondo, A., Ferrari, A., and Laloui, L. (2021). "Generalized effective stress concept for saturated active clays". Canadian Geotechnical Journal. 58(11), 1627-1639. 